\title{
SOFT TISSUE SARCOMAS OF EXTREMITIES - MRI ROLE IN THE DIAGNOSTIC EVALUATION
}

doi: $10.2478 /$ rojost-2018-0056

\author{
A.M. Bratu', I.A. Sălcianu' ${ }^{1}$, C. Zaharia ${ }^{1}$, G. lana ${ }^{2}$, A.N. Marinescu ${ }^{2}$ \\ ${ }^{1}$ Department of Radiology and Medical Imaging, "Colțea" Clinical Hospital, Bucharest, Romania; "Carol \\ Davila" University of Medicine and Pharmacy, Bucharest, Romania \\ ${ }^{2}$ Department of Radiology and Medical Imaging, University Emergency Hospital, Bucharest, Romania; "Carol \\ Davila" University of Medicine and Pharmacy, Bucharest, Romania
}

Introduction. Soft tissue sarcomas (STS) are rare entities of soft tissue cancers.

Their incidence is low, of only $1 \%$ of the malignant tumors.

In terms of localization, most of the STS affect the extremities, and their incidence is much higher in children than in adults.

Material and method. The present paper is a retrospective study that includes tumors with lower limb localizations, including the bony pelvis, over a 3-year period (2013-2016). The study group consisted of 29 patients who, following the MRI examination, were diagnosed with softtissue tumors. Of the 29 patients, 17 patients had a MRI (magnetic resonance imaging) and an anatomopathological diagnosis of leiomyosarcoma.

The location of the tumor, its characteristics, and the relationship with the adjacent anatomical structures were analyzed in all cases.

Results. The ages of the final group of 17 patients ranged between 28 and 84 years, with female predominance. In terms of localization, one showed a muscle tumor in the pelvis, namely left oblique muscle, other cases being located in the thigh and knee. A special importance was given to the superficial and profound location. In 5 cases, the tumor was localized in subcutaneous fatty tissue, thus superficial.

In terms of the contours of the tumor, well-defined margins were present in 11 cases, and poorly defined contour in 6 cases.

Regarding the size, the leiomyosarcomas in our study had dimensions between $5.2 \mathrm{~cm}$ and $18 \mathrm{~cm}$, and their structure was inhomogeneous, with the presence of necrosis and calcifications. Necrosis was found in 14 cases, and calcifications were present in $68 \%$, being more frequent than necrosis. Except for the necrotic areas, the contrast enhancement was intense.

Conclusions. Although the diagnosis is always histopathological, the MRI plays an important role in defining a precise localization and tumor characteristics.

Keywords: leiomyosarcoma, MRI, diagnosis 